\title{
Reduction PAPR of OFDM Signals by Combining SLM with DCT Transform
}

\author{
Zhongpeng Wang \\ School of Information and Electronic Engineering, Zhejiang University of Science and Technology, \\ Hangzhou, China \\ E-mail:wzp1966@sohu.com
}

Received July 20, 2010; revised August 31, 2010; accepted October 6, 2010

\begin{abstract}
One of main disadvantage of Orthogonal frequency division multiplexing (OFDM) is high peak-to-average power ratio (PAPR). In this paper, two effective PAPR reduction schemes are proposed. These techniques combine the DCT and SLM techniques. The scheme 1 is composed of the DCT followed by the SLM technique, and the DCT is used followed by conventional SLM in proposed scheme 2. Simulation results show that the proposed schemes can obtain significant PAPR reduction performance with that of ordinary SLM techniques.
\end{abstract}

Keywords: SLM, DCT Transform, PAPR, OFDM

\section{Introduction}

Orthogonal frequency division multiplexing (OFDM) has been recently seen rising popularity in wireless applications. For wireless communications, an OFDM-based system can provide greater immunity to multi-path fading and reduce the complexity of equalizers. Now OFDM have been included in digital audio/video broadcasting (DAB/DVB) standard in Europe, and IEEE 802.11, IEEE 802.16 wireless broadband access systems, etc.

However, OFDM also has its shortcoming. The major drawback of OFDM signal is its large peak-to-average power ratio (PAPR), which causes poor power efficiency or serious performance degradation to transmit power amplifier. To reduce the PAPR, many techniques have been proposed. Such as clipping, partial transmit sequence (PTS) [1], selected mapping (SLM) [2], interleaving, nonlinear companding transforms, hadamard transforms [3] and other techniques etc [4,5]. These schemes can mainly be categorized into signal scrambling techniques, such as PTS, and signal distortion techniques such as clipping. Among those PAPR reduction methods, the simplest scheme is to use the clipping process. However, using clipping processing causes both in-band distortion and out-of-band distortion. The bit error rate of system is degraded by using clipping. The SLM is relatively attractive since it can obtain better PAPR by modifying the OFDM signal without distortion. The main disadvantage of SLM is that its complexity is high. Now many extension schemes for reducing complexity of LSM have been presented [6,7]. In this paper, an efficient reducing PAPR technique based on joint SLM and DCT matrix transform is proposed.

The organization of this paper is as follow. Section 2 presents OFDM signal model and formulates the problem of PAPR. Section 3, the reduction PAPR schemes based on combined DCT transform matrix and SLM technique are proposed. Numerical results are presented in Section 4. Section 5 draws conclusions.

\section{OFDM Signal and Problem Formulation}

\subsection{OFDM Signal Model and PAPR Problem}

In this section, we review the basic of OFDM transmitter and the PAPAR definition. Consider an OFDM consisting of $N$ subcarriers. Let a block of $N$ symbol $\mathbf{X}=\left\{X_{k}, k=0,1, \cdots, N-1\right\}$ is formed with each symbol modulating one of a set of subcarriers. $\left\{f_{k}, k=\right.$ $0,1, \cdots, N-1\}$. The $N$ subcarriers are chosen to be orthogonal, that is, $f_{k}=k \Delta f$, where $\Delta f=1 /(N T)$ and $T$ is the original symbol period. Therefore, the complex baseband OFDM signal can be written as

$$
x(t)=\frac{1}{\sqrt{N}} \sum_{k=0}^{N-1} X_{k} e^{j 2 \pi \pi_{k} t}, \quad 0 \leq t \leq N T
$$

In general, the PAPR of OFDM signals $x(t)$ is de- 
fined as the ratio period between the maximum instantaneous power and its average power during an OFDM symbol

$$
\operatorname{PAPR}=\frac{\max _{0 \leq t \leq N T}\left[|x(t)|^{2}\right]}{1 /(N T) \int_{0}^{N T}|x(t)|^{2} d t}
$$

Reducing the max $|x(t)|$ is the principle goal of PAPR reduction techniques. In practice, most systems deal with a discrete-time signal, therefore, we have to sample the continuous-time signal $x(t)$.

To better approximate the PAPR of continuous-time OFDM signals, the OFDM signals samples are obtained by $L$ times oversamping. By sampling $x(t)$ defined in Equation (1), at frequency $f_{s}=L / T$, where $L$ is the oversampling factor, the discrete-time OFDM symbol can be written as

$$
x(n)=\frac{1}{\sqrt{N}} \sum_{k=0}^{N-1} X_{k} e^{j \frac{2 \pi}{N L} k n}, \quad 0 \leq n \leq N L-1
$$

Equation (3) can be implemented by using a length $(N L)$ IFFT operation. The new input vector $X$ is extended from original $X$ by using the so-called zero-padding scheme, i.e., by inserting $(L-1) N$ zeros in the middle of $X$. The PAPR computed form the $L$-times oversampled time domain OFDM signal samples can be defined as

$$
\operatorname{PAPR}[x(n)]=10 \lg \frac{\max _{0 \leq t \leq N L-1}\left[|x(n)|^{2}\right]}{E\left[|x(n)|^{2}\right]}
$$

However, the PAPR does not increase significantly after $L=4$. In order to avoid aliasing the out-of-band distortion into the data bearing tones and in order to accurately describe the PAPR an oversampling factor $L \geq 4$ is required.

We can evaluate the performance of PAPR using the cumulative distribution of PAPR of OFDM signal. The cumulative distribution function (CDF) is one of the most regularly used parameters, which is used to measure the efficiency of and PAPR technique. The CDF of the amplitude of a signal sample is given by

$$
F(z)=1-\exp (z)
$$

However, the complementary CDF (CCDF) is used instead of CDF, which helps us to measure the probability that the PAPR of a certain data block exceeds the given threshold. The CCDF of the PAPR of the data block is desired is our case to compare outputs of various reduction techniques. This is given by

$$
\begin{aligned}
P(P A P R>z) & =1-P(P A P R>z) \\
& =1-(F(z))^{N}=1-(1-\exp (-z))^{N}
\end{aligned}
$$

\subsection{Selected Mapping (SLM)}

The SLM technique was first described by Bauml et al. [3]. In the SLM, the input data sequences are multiplied by each of the phase sequences to generate alternative input symbol sequences. Each of these alternative input data sequences is made the IFFT operation, and then the one with the lowest PAPR is selected for transmission. Figure 1 shows the block of the SLM technique. $X$ is the OFDM data block, $B_{u}$ is the phase vectors and $X_{u}$ is the modified data vectors in the frequency domain. So the time domain signal

$$
x_{u}(t)=\frac{1}{\sqrt{N}} \sum_{k=0}^{k=N-1} X_{k} B_{u, k} e^{j 2 \pi k \Delta f t}, \quad 0 \leq t \leq N T
$$

where $u=1,2, \cdots, U$ and $N$ is length of $X$, also the number of sub-carriers.

Among the modified data blocks, the one with the lowest PAPR is selected for transmission. The amount of PAPR reduction for SLM depends on the number of phase sequences $U$ and the design of the phase sequences.

\subsection{DCT Transform}

The Discrete Cosine Transform (DCT) is a Fourier-like transform, which was first proposed by Ahmed et al. (1974) [8]. The idea to use the DCT transform is to reduce the autocorrelation of the input sequence to reduce the peak to average power problem and it requires no side information to be transmitted to the receiver. In the section, we briefly review DCT transform.

The formal definition of the DCT of one-dimensional of length $N$ is given by the following formula:

$$
\begin{aligned}
& X_{c}(k)=\alpha(k) \sum_{n=0}^{N-1} x(n) \cos \left[\frac{\pi(2 n+1) k}{2 N}\right] \\
& \text { for } \quad k=0 \quad \cdots \quad N-1
\end{aligned}
$$

Similarly, the inverse transformation is defined as

$$
\begin{aligned}
& x(n)=\alpha(k) \sum_{u=0}^{N-1} \alpha(k) X_{c}(k) \cos \left[\frac{\pi(2 n+1) k}{2 N}\right] \\
& \text { for } \quad n=0 \quad \cdots \quad N-1
\end{aligned}
$$

For both equations ( ) and ( ) $\alpha(u)$ is defined as

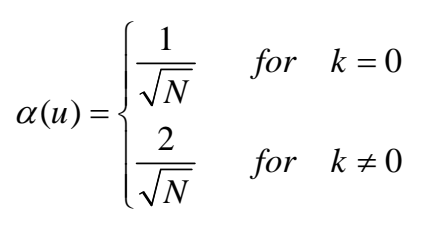

The equation ( ) is expressed in matrix below

$$
\mathrm{X}_{\mathrm{c}}=\mathrm{C}_{\mathrm{N}} \mathrm{X}
$$

where $\mathrm{X}_{\mathrm{c}}$ and $\mathbf{x}$ are both vector with $N \times 1$, and $\mathrm{C}_{N}$ 
is a DCT transform matrix with $N \times N$. The row (or column) of the DCT matrix $\mathrm{C}_{N}$ are orthogonal matrix vectors. Then we can use this property of the DCT matrix and reduce the peak power of OFDM signals.

\section{Proposed Schemes}

The main idea of the proposed scheme is to use a combination of two appropriate methods. One is the DCT matrix transform technique and the other is the SLM technique. The technique is similar to the scheme proposed in literature [6]. The transmitter block is showed in Figure 1. We call this scheme is scheme 1. In the transmit end, the data stream is firstly transformed by DCT matrix, then the transformed data is processed by the SLM unit. If data block passed by DCT matrix before IFFT, the autocorrelation coefficients of IFFT input is reduced, then the PAPR of OFDM signal could be reduced.

In this paper, we use DCT matrix after SLM to further reduce the PAPR of signal. We call this scheme as scheme 2. In his fashion, the autocorrelation of the signal, which has been processed by SLM, is reduced by DCT matrix transform. The PAPR of fine output signal is further reduced. The block of transmitter is showed in Figure 2.

\section{Simulation Results}

In this section, computer simulations are used to evaluate the peak-to-average ratio reduction capability with proposed scheme. The channel is modeled an additive while Gaussian noise (AWGN). In simulation, an OFDM system is considered with subcarrier $N=64$ and QPSK modulation. In SLM unit, U different random phase sequences are used.

The results of original SLM are given in Figure 3. We can see that the reduction effect is improved with the increasing of sub-block number $U$.

The results of proposed schemes 1 are given in Figure 4 and Figure 5 is the results of proposed scheme 2. From these figures, we see that our proposed scheme 1 and scheme 2 for QPSK have better performance than original SLM (Figure 3). When $U=2$ and CCDF $=10^{-3}$, the proposed scheme 1 may reduces PAPR about $1.8 \mathrm{~dB}$ and the proposed scheme 2 may reduces about $1.2 \mathrm{~dB}$.

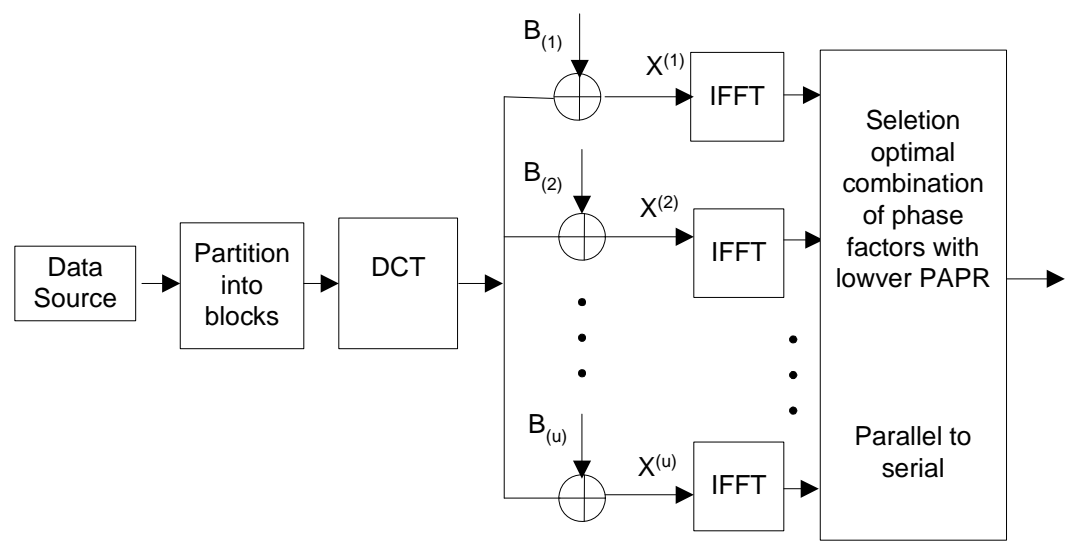

Figure 1. Diagram of proposed SLM scheme 1.

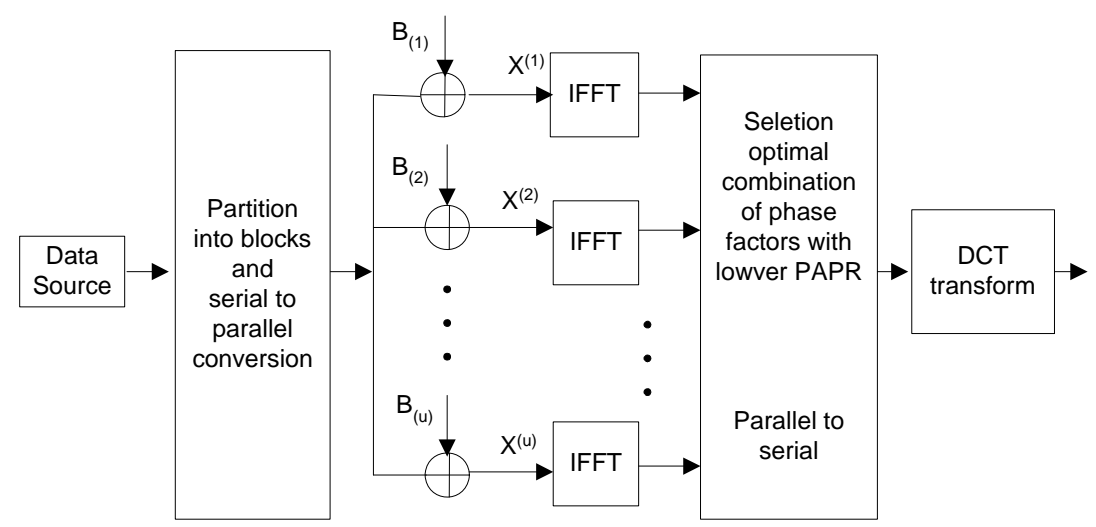

Figure 2. Diagram of proposed SLM scheme 2. 


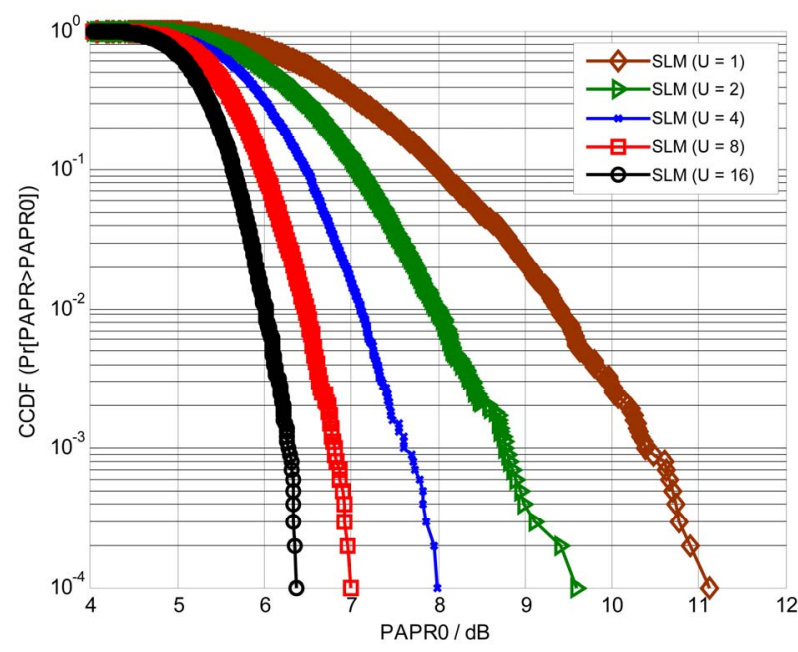

Figure 3. CCDF of original SLM.

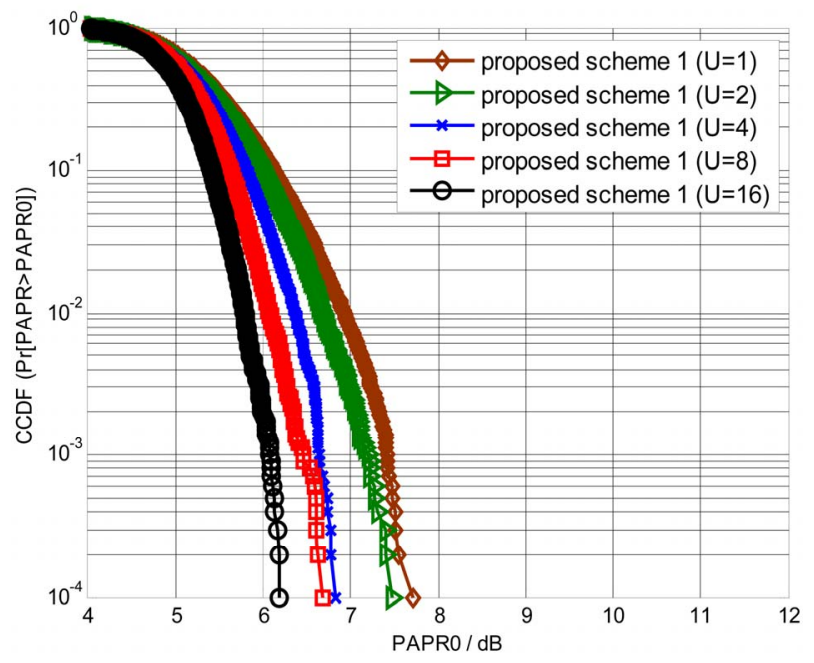

Figure 4. CCDF of proposed scheme 1.

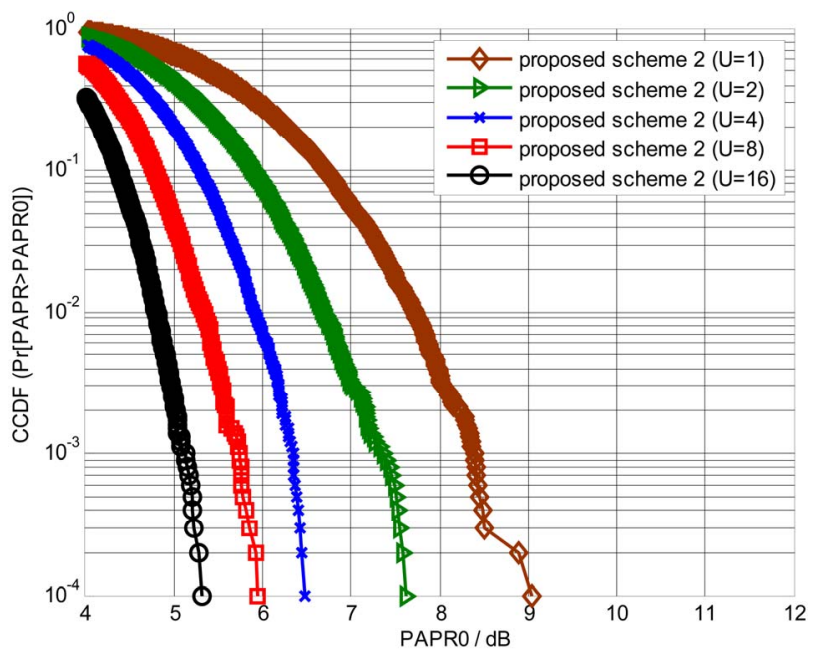

Figure 5. CCDF of proposed scheme 2.
Compared Figure 4 and Figure 5, we can see that the performances of proposed scheme 2 is better than the proposed scheme 1 when the increasing of sub-block number $U>4$. At $U=16$, the proposed scheme 2 may reduce about $1 \mathrm{~dB}$ compared with the proposed scheme 1 .

\section{Conclusion}

In this paper, techniques for PAPR reduction of OFDM signals have been proposed. These techniques combine the SLM technique and DCT transform. The scheme 1 is composed of DCT matrix transform followed by conventional SLM, while DCT transform is used before conventional SLM processing unit in proposed scheme 2 . The PAPR reduction performances are evaluated by computer simulation. Simulation results state that the PAPR reduction performance is greatly improved compared to conventional SLM. When sub-block number $U$ $=2$ and $\mathrm{CCDF}=10^{-3}$, the proposed scheme 1 may reduces PAPR about $1.8 \mathrm{~dB}$ and the proposed scheme 2 may reduces about $1.2 \mathrm{~dB}$.

\section{Acknowledgement}

The author would like to thank the reviewer for their detailed and useful suggestions that have helped the presentation of this paper.

\section{References}

[1] R. W. Bauml, R. F. Fischer and J. B. Huber, "Reducing the Peak-to-Average Power Ration of Multicarrier Modulation by Selected Mapping,” Electronics Letters, Vol. 32, No. 22, 1996, pp. 2050-2057.

[2] S. H. Muller and J. B. Huber, "OFDM with Reduced Peak to Average Power Ratio by Optimum Combination of Partial Transmit Sequences,” IEEE Electronics Letters, Vol. 33, No. 5, 1997, pp. 368-369.

[3] M. Park, J. Heeyong, J. Cho, N. Cho, D. Hong and C. Kang, "PAPR Reduction in OFDM Transmission Using Hadamard Transform,” IEEE International Conference on Communications, Vol. 1, June 2000, pp. 430-433.

[4] T. Jiang and Y. Wu, "An Overview: Peak-to-Average Power Ratio Reduction Techniques for OFDM Signals," IEEE Transactions on Broadcasting, Vol. 54, No. 2, June 2008, pp. 257-268.

[5] S. H. Han and J. H. Lee, “An Overview of Peak-to-Average Power Ratio Reduction Techniques for Multicarrier Transmission,” IEEE Transactions on Wireless Communications, Vol. 12, No. 2, April 2005, pp. 56-65.

[6] A. Zolghadrasli and M. H. Ghamat, "An Overview of PAPR Reduction Techniques for Multicarrier Transmission and Propose of New Techniques for PAPR Reduc- 
tion,” Iranian Journal of Electrical and Computer Engineering, Vol. 7, No. 2, Summer-Fall 2008, pp. 115-120.

[7] N. V. Irukulapati, V. K. Chaka and A. Jain, "SLM Based PAPR Reduction of OFDM Signal Using New Phase Sequence,” Electronics Letters, Vol. 45, No. 24, 2009, pp.
1231-1232.

[8] N. T. Ahmed, Natarajan and K. R. Rao, "Discrete Consine Transform," IEEE Transactions on Computer, Vol. 23, 1974, pp. 90-93. 